\title{
A randomised controlled trial of three or one breathing technique training sessions for breathlessness in people with malignant lung disease
}

Miriam J Johnson ${ }^{1 *}$, Mona Kanaan², Gerry Richardson ${ }^{3}$, Samantha Nabb ${ }^{4}$, David Torgerson², Anne English ${ }^{5,6}$, Rachael Barton ${ }^{7}$ and Sara Booth ${ }^{8,9}$

\begin{abstract}
Background: About $90 \%$ of patients with intra-thoracic malignancy experience breathlessness. Breathing training is helpful, but it is unknown whether repeated sessions are needed. The present study aims to test whether three sessions are better than one for breathlessness in this population.

Methods: This is a multi-centre randomised controlled non-blinded parallel arm trial. Participants were allocated to three sessions or single (1:2 ratio) using central computer-generated block randomisation by an independent Trials Unit and stratified for centre. The setting was respiratory, oncology or palliative care clinics at eight UK centres. Inclusion criteria were people with intrathoracic cancer and refractory breathlessness, expected prognosis $\geq 3$ months, and no prior experience of breathing training. The trial intervention was a complex breathlessness intervention (breathing training, anxiety management, relaxation, pacing, and prioritisation) delivered over three hour-long sessions at weekly intervals, or during a single hour-long session. The main primary outcome was worst breathlessness over the previous 24 hours ('worst'), by numerical rating scale $(0=$ none; $10=$ worst imaginable). Our primary analysis was area under the curve (AUC) 'worst' from baseline to 4 weeks. All analyses were by intention to treat.
\end{abstract}

Results: Between April 2011 and October 2013, 156 consenting participants were randomised (52 three; 104 single). Overall, the 'worst' score reduced from 6.81 (SD, 1.89) to $5.84(2.39)$. Primary analysis [ $=124$ (79\%)], showed no between-arm difference in the AUC: three sessions 22.86 (7.12) vs single session 22.58 (7.10); $P$ value $=0.83$ ); mean difference $0.2,95 \% \mathrm{Cls}$ (-2.31 to 2.97). Complete case analysis showed a non-significant reduction in QALYs with three sessions (mean difference $-0.006,95 \% \mathrm{Cls}-0.018$ to 0.006 ). Sensitivity analyses found similar results. The probability of the single session being cost-effective (threshold value of $£ 20,000$ per QALY) was over $80 \%$.

Conclusions: There was no evidence that three sessions conferred additional benefits, including cost-effectiveness, over one. A single session of breathing training seems appropriate and minimises patient burden.

Trial registration: Registry: ISRCTN; Trial registration number: ISRCTN49387307; http://www.isrctn.com/ISRCTN49387307; registration date: 25/01/2011

Keywords: Breathing training, Breathlessness, Dyspnoea, Cancer, Neoplasm

\footnotetext{
* Correspondence: miriam.johnson@hyms.ac.uk

'Hull York Medical School, Hertford Building, University of Hull, Hull HU6 7RX, UK

Full list of author information is available at the end of the article
} 


\section{Background}

Lung cancer is the most common cause of death from cancer worldwide, responsible for an estimated one in five (1.59 million deaths, $19.4 \%$ total deaths) [1]. Rarely curable, breathlessness is a problem in up to $90 \%$ of patients [2]. Breathlessness is harder to treat than pain and is a cause of emergency hospital admission [3, 4]. Complex non-pharmacological supportive care interventions for people with lung cancer appear to be beneficial and cost-effective, although which intervention components are the most beneficial is less clear [5-11]. Four phase III trials have compared a complex breathlessness intervention with standard care [7, 9-11], two using intensity of breathlessness as the primary outcome [7,9], one using the mastery subscale of the Chronic Respiratory Questionnaire (CRQ) [11], and one using patient distress due to breathlessness (numerical rating scale (NRS) 0-10) [10]. All trials demonstrated clinically and statistically significant benefit with the intervention.

The National Institute for Health and Clinical Excellence (NICE) guidance for Lung Cancer recommends access to these treatments [12], although there is a need for further research regarding optimum service delivery, particularly for cancer patients where the evidence base is less strong than for rehabilitation programmes in non-malignant cardiorespiratory conditions $[13,14]$. A review of exercise-based training for people with non-small cell lung cancer concluded that there was some benefit for exercise tolerance (often limited by breathlessness), but these studies focussed on people having surgery, rather than those living with lung cancer [15].

The systematic implementation of breathing training services for people with cancer in practice is challenging [16]. Visits to or by clinicians may be onerous to patients with a poor performance status and progressive disease, such as those with lung cancer, even if provided in their own home. However, many patients have no training at all or receive a single ad hoc session only; it is unknown whether such single sessions are effective.

The primary hypothesis was that three sessions are superior to one, in terms of worst breathlessness intensity over the previous 24 hours in people with intrathoracic cancer. The primary objective was to assess the effectiveness of these two modes of delivery with regard to the relief of breathlessness intensity. We anticipated that three sessions of breathing training would have greater benefit than one.

Secondary objectives were to test which mode was more effective for other aspects of breathlessness, function, quality of life, psychological distress and coping, and cost-effectiveness.

\section{Methods}

Trial design

This multi-centre pragmatic randomised controlled nonblinded parallel arm trial assessed the effect of breathing training delivered over three 1 hour-long sessions at weekly intervals, or during a single session in the management of patients with refractory breathlessness due to intrathoracic cancer.

\section{Participants and setting}

Patients from eight centres in England, Scotland, and Wales attending hospital respiratory, oncology, palliative care clinics or hospices were screened by the research nurse in conjunction with the patients' usual clinical team. Adults with intra-thoracic malignancy (primary or secondary tumours) were eligible. Participants could be randomised to trial intervention if they had refractory breathlessness with a self-reported intensity of $\geq 3 / 10$ on a NRS, where $0=$ no breathlessness and 10 = worst imaginable breathlessness, and a clinicianestimated prognosis of at least 3 months. Participants with breathlessness intensity of $<3 / 10$ were followed-up at monthly intervals to assess eligibility for randomisation. Refractory breathlessness was defined as persistent breathlessness despite treatment of reversible causes [17]. Patients were excluded if they had intercurrent illness or co-morbidities making completion of the trial unlikely, worsening breathlessness requiring urgent medical intervention, or prior breathing training.

Centres were eligible if clinicians provided breathing training and were willing to deliver the trial intervention. The intervention could be delivered in the hospital, hospice, or a patient's home according to patient choice.

The protocol, procedures, and trial documentation were approved by the independent UK Integrated Research Approval System via the Sheffield Research Ethics Committee (ref 10/H1308/66). Subsequent Research and Development NHS governance approval was obtained for all sites prior to recruitment. The trial was registered (ISRCTN49387307) and the protocol followed CONSORT recommendations. All participants gave written informed consent.

\section{Intervention and comparator}

All participants received training in four techniques (Box 1) during an hour-long session, supported by written and DVD/video reinforcement material and a telephone call from their therapist a week after the last session. Those randomised to the three session arm received two further hour-long clinic sessions at weeks 2 and 3 to reinforce and practice the techniques. Training was provided by the usual therapist providing the service. 


\section{Box 1. Breathing management techniques}

\section{Breathing control}

The patient sits comfortably with their back supported, the shoulders relaxed, and the upper chest remaining as still as possible. The patient places their hand in front of the lower ribs and upper abdomen, and is asked to breathe in and then out, taking longer than the time taken to breathe in.

\section{Pacing/prioritising}

The patient is taught to control their breathing when walking up stairs or on level ground. They are instructed to breathe in time with the steps taken, e.g. breathe in for one step and then breathe out for two. The patient is instructed to find the rhythm that suits them best. Participants define their priorities regarding daily activity which is limited by dyspnoea. Goals are set, and a plan discussed with the therapist on how they may be achieved.

\section{Relaxation}

The patient is instructed in progressive muscle relaxation using a standard script and given a study-specific CD to practise at home.

\section{Anxiety management}

Patients are instructed to use a tool called The Calming Hand

[39]. Patients are asked to recall instructions when they

experience anxiety or panic. Each instruction is related to a digit

on their hand: thumb, recognise breathing related anxiety; index

finger, sigh out; third finger, inhale slowly; fourth finger, exhale

slowly; little finger, relax hands, stretch and stop.

The four techniques of breathlessness training were standardised prior to trial commencement, but different centres could provide local additional non-pharmacological therapies such as exercise if this was a usual part of their service. The type of professional (e.g. nurse, physiotherapist, occupational therapist) was not stipulated.

\section{Outcomes}

The primary outcome measure, defined by previous feasibility work [18], was patient-reported intensity of the worst breathlessness over the past 24 hours ('worst'; 0-10 numerical rating scale [NRS], $0=$ no breathlessness; $10=$ the worst imaginable breathlessness) $[19,20]$.

Secondary measures included average intensity of breathlessness over the past 24 hours ('average'); distress due to breathlessness and 0-10 NRS coping with breathlessness ('distress') using NRS 0-10 scales anchored with $0=$ none and $10=$ worst; 'coping' and satisfaction with care of breathlessness (using 0-10 NRS scales anchored with $0=$ none and 10 = best outcome); injustice and catastrophizing scale; quality of life Chronic Respiratory Questionnaire - Self-Administered-Survey (CRQ-SAS) [21]; Hospital Anxiety and Depression Scale (HADS) [22]; Karnofsky performance scale (KPS), [23], health status (EQ-5D and EQ-visual analogue scale (EQVAS)) [24]; coping (BriefCOPE) [25]; and global impression of change and health service utilisation.

Baseline assessments included demographic data (age, sex, smoking history), KPS, medication and personality aspects (Big Five Inventory (BFI) [26]; Mental Toughness Questionnaire (MTQ)), and pre-randomisation preference for trial allocation. Follow-up assessments were at weeks 1, 2, 3, 4, and 8 after the first training session. All outcome measures were assessed at baseline, week 4 , and week 8 except the Big Five Inventory and Mental Toughness Questionnaire. At weeks 1-3 only NRS scores, EQ-5D and EQVAS were measured. Adverse events were noted at each contact. The primary analysis point was week 4 . After week 8 participants had an optional monthly telephone call from the trial nurse to record NRS intensity until withdrawal or death.

\section{Sample size}

Using data from previous feasibility work [18] to detect a difference of $30 \%$ in area under the curve (AUC) for NRS worst breathlessness (standard deviation (SD), 2.6) at 4 weeks at $80 \%$ power and $5 \%$ two-tailed statistical significance, with a 2:1 randomisation, allowing for $30 \%$ attrition, a sample size of 146 participants was needed. Although week 4 attrition in the feasibility trial was $50 \%$, a reduction to $30 \%$ was anticipated with earlier consent (consent to trial participation even if their reported breathlessness intensity did not fulfil the criteria for randomisation). AUC was chosen as more indicative of the experience of breathlessness over the trial than change in NRS scores, or comparison of week 4 NRS scores as it includes measures at weekly time points.

\section{Sample size re-calculation}

A review of week 4 attrition (June 2013) showed nearly $30 \%$ drop out. In order to reduce the risk of loss of power, a further 10 participants were recruited.

\section{Randomisation and masking}

Online 3- or 6-block randomisation (central computer generated sequence) and allocation of consenting participants was performed by the York Trials Unit using a 1:2 ratio (1 three: 2 single). This was done in order to minimise a potential increase in demand on usual services from participating in a trial actively seeking to recruit patients. As this was a pragmatic trial, centres maintained their usual practice other than providing the intervention standard components. To account for site differences in cancer therapies and additional breathlessness measures, participants were stratified by centre. Initially, stratification for 
site of first intervention (home or clinic) was planned, but as this would not always be known at randomisation, this was not done. Due to the likelihood of participants inadvertently disclosing their allocation arm to the research nurse, allocation was not blinded [18]. Trial assessments were conducted by research nurses.

\section{Statistical methods}

Baseline characteristics are described and presented in tabular form for all participants and presented by whether they were lost to follow-up or remained in the trial. Mean and SD are presented for quantitative data and number and percentage for categorical data. Summary statistics over time are also reported for the repeated outcome measures as are missing data.

For the primary analysis of AUC at 4 weeks (worst breathlessness), we used a two-sample t-test to compare the two arms. Multiple regression was used to estimate the effect of three sessions compared to one adjusting for baseline characteristics (age, sex, baseline breathlessness intensity, and smoking status). To account for missing data we i) utilised multiple imputation method for the primary outcome and ii) used complete cases for all secondary measures. Variation resulting from different practices at different sites was accounted for in the analysis by the stratification [27]. Management of missing data for the cost-effectiveness analysis is described below.

\section{Cost-effectiveness analysis}

The cost-effectiveness of three sessions versus one was compared. Costs were estimated for both arms and included the cost of the intervention and other health-related resource use costs. The effectiveness measure for this analysis was the Quality Adjusted Life Year (QALY) generated from responses to the EQ5D. A sensitivity analysis using multiple imputation to account for missing data was performed.

\section{Results}

Between April 2011 and October 2013, 156 participants were randomised (52 to three sessions; 104 to single session). Two withdrew from each arm prior to the intervention and were excluded from the analysis. Data for the AUC from baseline to week 4 for the primary outcome was available for 124 (79 \%) participants. Participant flow throughout the trial, including reasons for withdrawal, can be seen in Fig. 1.

\section{Trial setting and implementation}

In this pragmatic trial, the intervention was delivered in a variety of settings and by different therapists (Table 1 ). All four techniques were documented in the clinical record. In the single arm, 91 (89\%) received one session of all four techniques. In the three session arm, 39 (78 \%) received three sessions of all the techniques.

\section{Trial population}

Baseline characteristics between the two arms were similar apart from a clinically important difference in the mastery domain of the CRQ-SAS (three 3.9 [SD, 1.18]; single 4.48 [1.17]) and anxiety (HADS three 7.62 [4.34]; single 6.12 [4.19]; Table 2). For the group as a whole, the average age was 69.4 [9.35] years and $40 \%$ were women. 'Worst' and 'average' breathlessness intensity was 6.81 [1.89] and 5.53 [1.68], respectively; 133 (88.6\%) had a primary lung carcinoma, 12 (8 \%) had mesothelioma, and 5 (3.3 \%) had an extrathoracic primary site. Lung metastases were present in 23 (17\%). The average KPS was $70.6 \%$ [9.54]. Most were exsmokers (114 [76 \%]) or current smokers (23 [15\%]). Baseline measures were similar for pre-randomisation preference for trial allocation, mood, personality, or coping approaches, which might have influenced engagement with the intervention.

\section{Primary outcome}

Overall, the 'worst' score reduced from 6.81 (SD, 1.89) at baseline to 5.84 (2.39) at week 4 . There was no betweenarm difference in the AUC (cubic): three sessions 22.86 (7.12) versus single session 22.58 (7.10); $P$ value $=0.83$; mean difference $0.2,95 \%$ CIs (-2.31 to 2.97 ).

\section{Secondary outcomes}

There was no evidence of a between-arm difference in the AUC for 'average' and 'coping' although both arms improved by week 4 for all domains of the CRQ-SAS. However, in the three session arm, both distress from breathlessness (AUC 16.23 [7.17] three vs 12.29 [8.27] single; $P=0.01)$ and sense of mastery over breathlessness (4.44 [1.25] three vs 5.03 [1.15] single; $P=0.02$ ) were worse.

Adjusting for baseline variables (Table 3), baseline breathlessness intensity strongly predicted the AUC; greater with worse baseline breathlessness $(P=0.001)$. A consistent pattern, although not statistically significant, was seen for current smoker status: associated with worse AUC for 'worst' (co-efficient 4.06; $95 \%-1.36$ to 9.49), 'average' (4.67; 0.11 to 9.24$)$, 'distress' (5.13; -0.75 to 11.0$)$, 'coping' (-5.11; CIs -10.7 to 0.53$)$, and 'satisfaction' $(-7.66 ;-14$ to -1.35$)$ compared with never smokers; these estimates were higher than those associated with ex-smoker status ('worst' $3.15,-0.43$ to 7.74 ; 'average' $2.82,-1.08$ to 6.73 ; 'distress' $2.98,-1.95$ to 7.91 ; 'cope' $-4.84,-9.64$ to -0.04 ; 'satisfaction' $-5.17,-10.4$ to $0.04)$ compared to never smokers. Descriptive statistics for the summary weekly measures at weeks 4 and 8 are shown in Table 4. Outcomes relating to perception of or response to breathlessness/general condition show a consistent pattern of improvement over time, although less so for the fatigue domain of the CRQ-SAS. 


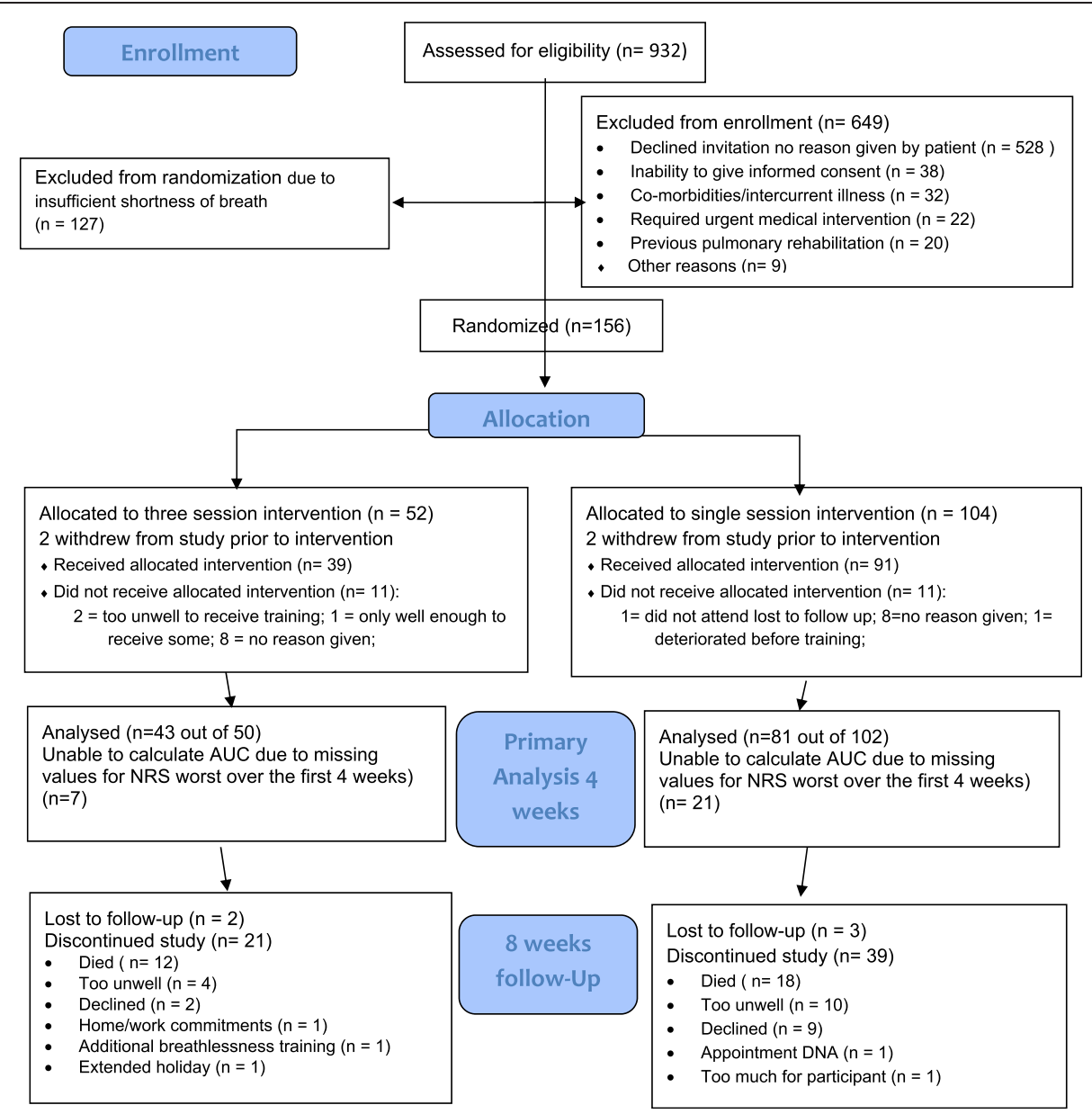

Fig. 1 CONSORT 2010 flow diagram

\section{Cost-effectiveness}

Complete case analysis showed a small non-significant reduction in overall QALYs with three sessions (mean difference $-0.006,95 \%$ CIs -0.018 to 0.006 ). These results were confirmed in a sensitivity analysis using multiple imputation of data (mean difference in QALYs $-0.008,95 \%$ $\mathrm{CI}-0.022$ to 0.006 ). Complete case analysis also showed a small non-significant increase in costs associated with three sessions. Thus, the single session would be considered dominant (improved outcomes at lower cost). The probability of the single session being cost-effective at a threshold value of $£ 20,000$ per QALY was over $80 \%$.

There was no evidence that the additional cost of three sessions is offset by lower resource use elsewhere and three sessions is associated with a worse QALY profile. Therefore, the single session intervention is likely to be cost-effective.

\section{Sensitivity analyses}

The sensitivity analyses using complete cases for secondary outcomes did not show any material difference.
Likewise, the analysis using multiple imputation for the primary outcome measure failed to demonstrate any significant difference.

A post hoc sensitivity analysis was performed in view of the clinically significant difference in baseline CRQ mastery domain and HADS anxiety in order to adjust for this difference, but did not materially affect the results (Additional file 1).

\section{Harms}

Overall, 50/156 (32\%) participants experienced 58 serious adverse events (deaths (30) or hospitalisation (28)). All were unrelated to the intervention and were expected events in the context of progressive cancer/comorbidities, apart from two (one in each allocated arm) which were unrelated to the intervention, but were unexpected (a fractured neck of femur following a fall and a femoral artery embolus).

\section{Discussion}

There was a clinically significant improvement, equating to a moderate effect [28], in breathlessness intensity and 
Table 1 Implementation of intervention in centres

\begin{tabular}{|c|c|c|c|}
\hline Centre/Site principal investigator & Setting & Breathing training provided by & Recruited \\
\hline $\begin{array}{l}\text { Cambridge University NHS } \\
\text { Hospitals Foundation Trust }\end{array}$ & Hospital & $\begin{array}{l}\text { Physiotherapist or Occupational Therapist: specialist } \\
\text { palliative care breathlessness intervention service }\end{array}$ & 12 \\
\hline \multicolumn{4}{|l|}{ Dr Sara Booth } \\
\hline $\begin{array}{l}\text { NHS Wales Cardiff and Vale University } \\
\text { Health Board and Velindre NHS Trust }\end{array}$ & \multirow[t]{2}{*}{$\begin{array}{l}\text { Tertiary oncology service } \\
\text { and palliative care clinics }\end{array}$} & \multirow[t]{2}{*}{ Physiotherapist } & \multirow[t]{2}{*}{24} \\
\hline Dr Anthony Byrne & & & \\
\hline $\begin{array}{l}\text { University Hospitals Coventry } \\
\text { and Warwickshire NHS Trust }\end{array}$ & \multirow[t]{2}{*}{ Cancer centre oncology clinics } & \multirow[t]{2}{*}{ Lung cancer clinical nurse specialists } & \multirow[t]{2}{*}{15} \\
\hline Dr Alison Franks & & & \\
\hline East Kent University Hospitals NHS Trust & \multirow[t]{2}{*}{ Hospice units } & \multirow[t]{2}{*}{ Physiotherapist } & \multirow[t]{2}{*}{28} \\
\hline Dr Andrew Thorns & & & \\
\hline NHS Lothian & \multirow[t]{2}{*}{ Oncology and palliative care clinics } & \multirow[t]{2}{*}{ Physiotherapist } & \multirow[t]{2}{*}{2} \\
\hline Prof Marie Fallon & & & \\
\hline NHS Greater Glasgow and Clyde & \multirow[t]{2}{*}{ Tertiary lung cancer clinic } & \multirow[t]{2}{*}{ Physiotherapist } & \multirow[t]{2}{*}{25} \\
\hline Dr Noelle O'Rourke & & & \\
\hline $\begin{array}{l}\text { Hull and East Yorkshire Hospitals NHS } \\
\text { Trust and Dove House Hospice, Hull }\end{array}$ & \multirow[t]{2}{*}{$\begin{array}{l}\text { Tertiary oncology service and } \\
\text { breathlessness intervention service }\end{array}$} & \multirow{2}{*}{$\begin{array}{l}\text { Physiotherapist: hospital and hospice based } \\
\text { specialist palliative care breathlessness } \\
\text { intervention service }\end{array}$} & \multirow[t]{2}{*}{33} \\
\hline Dr Rachael Barton & & & \\
\hline Dartford and Gravesham NHS Trust & \multirow{3}{*}{$\begin{array}{l}\text { Hospice units and district general } \\
\text { hospital oncology clinics }\end{array}$} & \multirow[t]{3}{*}{ Physiotherapist } & \multirow[t]{2}{*}{17} \\
\hline Dr Burhan Khan & & & \\
\hline & & & 156 \\
\hline
\end{tabular}

several other breathlessness measures in both arms and consistent with the benefit identified in usual care controlled trials [7, 9-11]. However, there was no evidence that three sessions conferred greater benefit than a single session for any of the outcomes. Furthermore, although not statistically significant, there was a greater reduction in health status in the three session arm compared with those receiving only one.

In both arms, improvements relating to the perception of breathlessness and response to living with it (intensity of breathlessness, distress due to, coping with and satisfaction of care of breathlessness; dyspnoea and mastery CRQ-SAS, anxiety, depression, sense of injustice and catastrophizing) were seen. This is consistent with the model of breathlessness as having two recognisable components, namely perception and emotional response [29], which can be reported by the patient [30] and has a physiological basis demonstrable by neuroimaging [31]. Measures which are more likely to represent underlying pathological processes such as the fatigue domain and KPS, either improved very little or deteriorated.

The prognosis for lung cancer remains poor for those unsuitable for surgery or other radical treatment and breathlessness is a feature of advanced rather than early stage cancer [32]. In our trial, participants were required to have at least moderate breathlessness $(>3 / 10$ NRS) to be randomised. Although the average performance status at baseline was $71 \%$, 30 (19\%) had died and 14 (8\%) were too unwell to provide data by 4 weeks, consistent with breathlessness as a sign of poor outcome.

The burden of unmet psychological and daily living needs in people with lung cancer is high [33], leading to calls for attention to palliative care needs, in the light of effective interventions [34] and evidence that palliative care reduces inappropriate and costly healthcare interventions [35]. A trial of early palliative care involvement in people with non-small cell lung cancer demonstrated improvement in quality of life and mood in conjunction with improved survival and less aggressive care at the end of life [36]. Two recently reported 'fast track' trials, which included breathlessness management interventions, confirmed benefit for those randomised to early rather than late palliative care $[10,11]$.

The economic and clinical burden of lung cancer treatment has been highlighted [37], but less is known about the burden of palliative care. The burden of nonpharmacological interventions should not be assumed to be negligible for people with advancing disease although reported patient and carer experience suggests that a comprehensive complex intervention is valued $[10,11]$. However, just as the optimum dose of cancer treatment is assessed for net-benefit, so palliative care interventions should be given the same consideration. It is of concern that distress due to breathlessness was rated 
Table 2 Baseline characteristics

\begin{tabular}{|c|c|c|c|c|c|c|c|}
\hline \multirow[b]{2}{*}{ Variable } & \multicolumn{3}{|l|}{ All participants } & \multicolumn{2}{|c|}{ Participants lost } & \multicolumn{2}{|c|}{ Remaining participants } \\
\hline & Three sessions & Single session & Total & Three & Single & Three & Single \\
\hline N & 52 & 104 & 156 & 9 & 23 & 43 & 81 \\
\hline Withdrew at baseline & 2 & 2 & 4 & & & & \\
\hline \multicolumn{8}{|l|}{ Age in years: } \\
\hline mean (SD [MS] & $68(11)[0]$ & 69 (9) [1] & 69 (9) [1] & 67 (8) [0] & $68(8)[1]$ & $69(11)[0]$ & $70(9)[0]$ \\
\hline $\min , \max$ & 38,87 & 47,92 & 38,92 & 53,77 & 55,83 & 38,87 & 47,92 \\
\hline \multicolumn{8}{|l|}{ Sex: N (\%) [MS] } \\
\hline Female & $21(43)[1]$ & 39 (39) [2] & $60(40)[3]$ & $1(11)[0]$ & $6(29)[2]$ & $21(50)[1]$ & $33(41)[0]$ \\
\hline Intrathoracic tumour: N (\%) & [0 MS] & [2 MS] & {$[2 \mathrm{MS}]$} & {$[0 \mathrm{MS}]$} & [2 MS] & [0 MS] & [0 MS] \\
\hline Non-small cell & $14(28)$ & $21(21)$ & $35(23.3)$ & $4(44)$ & $5(24)$ & $11(26)$ & $17(21)$ \\
\hline Small cell & $1(2)$ & $4(4)$ & $5(3.3)$ & & $2(9)$ & $1(2)$ & $2(2)$ \\
\hline Lung (histology not stated) & $30(60)$ & $63(63)$ & $93(62)$ & $5(56)$ & $13(62)$ & $26(60)$ & $51(63)$ \\
\hline Mesothelioma & $4(8)$ & $8(8)$ & $12(8)$ & & $1(5)$ & $4(9)$ & $7(9)$ \\
\hline Other & $1(2)$ & $4(4)$ & $5(3.3)$ & & & $1(2)$ & $4(5)$ \\
\hline \multicolumn{8}{|l|}{ Metastases: N (\%) [MS] } \\
\hline Yes & 9 (19) [2] & $14(16)[12]$ & $23(17)[14]$ & $1(14)[2]$ & 5 (29) [6] & $8(19)[1]$ & 9 (12) [6] \\
\hline Preference: N (\%) & [0 MS] & [1 MS] & [1 MS] & [0 MS] & [1 MS] & [0 MS] & [0 MS] \\
\hline High & $14(28)$ & $20(20)$ & $34(22.5)$ & $1(11)$ & $3(14)$ & $13(30)$ & $17(21)$ \\
\hline Low & $8(16)$ & $26(26)$ & $34(22.5)$ & $3(33)$ & $5(23)$ & $6(14)$ & $22(27)$ \\
\hline No preference & $28(56)$ & $55(54)$ & $83(55)$ & $5(56)$ & $14(64)$ & $24(56)$ & $42(52)$ \\
\hline Smoking: N (\%) & [0 MS] & [2 MS] & [2 MS] & [1 MS] & [1 MS] & [0 MS] & [1 MS] \\
\hline Never & $6(12)$ & $7(7)$ & $13(9)$ & & & $6(14)$ & $7(9)$ \\
\hline Current & $7(14)$ & $16(16)$ & $23(15)$ & $2(25)$ & $3(14)$ & $5(12)$ & $13(16)$ \\
\hline Ex-smoker & $37(74)$ & $77(77)$ & $114(76)$ & $6(75)$ & $19(86)$ & $32(74)$ & $60(75)$ \\
\hline \multicolumn{8}{|l|}{ Karnofsky } \\
\hline Mean (SD) [MS] & $70.6(9.0)[3]$ & 70.6 (9.9) [7] & 70.6 (9.5) [10] & $62.5(8.9)[1]$ & $63.8(13.2)[2]$ & $71.7(8.6)[2]$ & $72.2(7.9)[5]$ \\
\hline \multicolumn{8}{|l|}{$\begin{array}{l}\text { Breathlessness scores NRS: } \\
\text { mean (SD) [MS] }\end{array}$} \\
\hline Worst & $6.7(2.0)[6]$ & $6.9(1.9)[12]$ & $6.8(1.9)[18]$ & $7.6(1.8)[1]$ & $7.4(1.8)[3]$ & $6.6(2.0)[6]$ & $6.7(1.9)[10]$ \\
\hline Average & $5.4(1.8)[6]$ & $5.6(1.6)[13]$ & $5.5(1.7)[19]$ & $6.0(1.3)[1]$ & $6.6(2.0)[3]$ & $5.3(1.9)[6]$ & $5.3(1.4)[11]$ \\
\hline Distress & $4.7(2.8)[7]$ & $4.6(3.1)[12]$ & 4.6 (3.0) [19] & $4.5(2.3)[1]$ & $5.7(3.0)[3]$ & $4.7(2.8)[7]$ & $4.3(3.1)[10]$ \\
\hline Cope & $6.4(2.1)[6]$ & $7.0(2.1)[12]$ & $6.8(2.1)[18]$ & $6.3(2.4)[1]$ & $6.3(2.1)[3]$ & $6.5(2.0)[6]$ & $7.2(2.1)[10]$ \\
\hline Care provided & $6.3(3.4)[9]$ & 6.5 (3.4) [20] & 6.5 (3.4) [29] & $5.6(2.8)[2]$ & $6.0(3.6)[4]$ & $6.3(3.6)[8]$ & $6.8(3.3)[17]$ \\
\hline \multicolumn{8}{|l|}{$\begin{array}{l}\text { Chronic Respiratory Questionnaire - } \\
\text { SAS: mean (SD) [MS] }\end{array}$} \\
\hline Dyspnoea domain & $4.41(1.25)[0]$ & $4.38(1.31)[1]$ & 4.39 (1.30) [1] & $3.95(1.32)[1]$ & $4.38(1.43)[1]$ & $4.50(1.22)[0]$ & $4.38(1.27)[0]$ \\
\hline Fatigue & $3.02(0.95)[0]$ & 3.32 (1.11) [2] & 3.22 (1.07) [2] & $2.72(0.81)[1]$ & $2.94(1.14)[2]$ & $3.06(0.97)[0]$ & $3.41(1.08)[0]$ \\
\hline Emotional function & $4.17(1.10)[0]$ & $4.50(1.28)[2]$ & $4.39(1.23)[2]$ & $3.54(1.12)[1]$ & $3.81(1.38)[2]$ & $4.26(1.09)[0]$ & $4.68(1.17)[0]$ \\
\hline Mastery & $3.90(1.19)[0]$ & $4.48(1.17)[2]$ & $4.29(1.20)[2]$ & $2.94(0.50)[1]$ & $3.78(1.35)[2]$ & $4.06(1.19)[0]$ & $4.66(1.03)[0]$ \\
\hline \multicolumn{8}{|l|}{$\begin{array}{l}\text { Hospital Anxiety and Depression } \\
\text { Scale (HADS): mean (SD) [MS] }\end{array}$} \\
\hline Anxiety & $7.6(4.3)[0]$ & $6.1(4.4)[4]$ & $6.6(4.3)[4]$ & $8.5(4.8)[1]$ & $8.4(5.0)[3]$ & $7.6(4.3)[0]$ & $5.6(3.7)[1]$ \\
\hline Depression & $6.6(3.0)[0]$ & $6.2(3.9)[2]$ & $6.3(3.6)[2]$ & $8.1(2.2)[1]$ & $7.9(4.9)[2]$ & $6.4(3.1)[0]$ & $5.8(3.5)[0]$ \\
\hline Big Five Inventory (BFI): [MS] mean (SD) & {$[0]$} & [4] & {$[4]$} & [1] & [4] & {$[0]$} & [1] \\
\hline Extraversion & $3.06(0.75)$ & $3.30(0.83)$ & $3.22(0.81)$ & $3.11(0.94)$ & $3.00(0.78)$ & $3.05(0.71)$ & $3.37(0.82)$ \\
\hline
\end{tabular}


Table 2 Baseline characteristics (Continued)

\begin{tabular}{|c|c|c|c|c|c|c|c|}
\hline Agreeableness & $4.22(0.53)$ & $4.16(0.64)$ & $4.18(0.60)$ & $3.96(0.65)$ & $3.99(0.91)$ & $4.26(0.49)$ & $4.20(0.55)$ \\
\hline Conscientiousness & $4.00(0.64)$ & $4.11(0.61)$ & $4.07(0.62)$ & $3.90(0.60)$ & $3.94(0.69)$ & $4.00(0.66)$ & $4.16(0.59)$ \\
\hline Neuroticism & $2.64(0.83)$ & $2.51(0.88)$ & $2.55(0.86)$ & $3.01(0.89)$ & $2.93(1.08)$ & $2.60(0.82)$ & $2.40(0.80)$ \\
\hline Openness & $3.32(0.75)$ & $3.30(0.70)$ & $3.31(0.71)$ & $3.09(0.81)$ & $3.11(0.81)$ & $3.34(0.75)$ & $3.34(0.67)$ \\
\hline $\begin{array}{l}\text { Mental Toughness Questionnaire } \\
\text { (MTQ48): [MS] mean (SD) }\end{array}$ & {$[1]$} & {$[4]$} & {$[5]$} & [3] & {$[5]$} & {$[0]$} & {$[0]$} \\
\hline Challenge & $3.7(0.7)$ & $3.8(0.7)$ & $3.78(0.7)$ & $3.7(0.7)$ & $3.7(0.8)$ & $3.7(0.7)$ & $3.8(0.6)$ \\
\hline Commitment & $3.6(0.7)$ & $3.8(0.6)$ & $3.7(0.6)$ & $3.5(0.7)$ & $3.5(0.8)$ & $3.6(0.7)$ & $3.8(0.6)$ \\
\hline Control emotion & $3.3(0.6)$ & $3.3(0.6)$ & $3.3(0.6)$ & $3.3(0.4)$ & $3.1(0.8)$ & $3.25(0.7)$ & $3.3(0.6)$ \\
\hline Control life & $3.6(0.7)$ & $3.7(0.7)$ & $3.7(0.7)$ & $3.7(0.9)$ & $3.5(0.8)$ & $3.59(0.7)$ & $3.77(0.6)$ \\
\hline Confidence abilities & $3.6(0.6)$ & $3.7(0.6)$ & $3.7(0.6)$ & $3.7(0.5)$ & $3.5(0.8)$ & $3.6(0.6)$ & $3.68(0.6)$ \\
\hline Confidence interpersonal & $3.72(0.82)$ & $3.84(0.75)$ & $3.80(0.77)$ & $4.12(0.99)$ & $3.83(0.66)$ & $3.67(0.79)$ & $3.83(0.78)$ \\
\hline $\begin{array}{l}\text { Pre-randomisation patient } \\
\text { stated preference for study } \\
\text { allocation: [MS] N (\%) }\end{array}$ & {$[0]$} & {$[1]$} & {$[1]$} & {$[0]$} & {$[1]$} & {$[0]$} & {$[0]$} \\
\hline Three sessions & $14(28)$ & $20(20)$ & $34(22.5)$ & $1(11)$ & $3(14)$ & $13(30)$ & $17(21)$ \\
\hline Single session & $8(16)$ & $26(26)$ & $34(22.5)$ & $3(33)$ & $5(23)$ & $6(14)$ & $22(27)$ \\
\hline No preference & $28(56)$ & $55(54)$ & $83(55)$ & $5(56)$ & $14(64)$ & $24(56)$ & $42(52)$ \\
\hline
\end{tabular}

${ }^{* *}$ Adjusted for baseline breathlessness intensity, gender, smoking status, and age; Descriptive statistics of the primary and secondary breathlessness outcome measures, $P$ values and unadjusted and adjusted confidence intervals for the difference between the two arms

$\mathrm{N}=$ number SAS, Self-administered survey (supported by research nurse); SD, Standard deviation; AUC, Area under the curve. MS = Missing

worse in those receiving three sessions. It is interesting to note that those who withdrew from the trial had a lower performance status. If patients are taught how to address their breathlessness themselves in a single session and then allowed to see how they manage without repeat visits (with their inherent logistic challenges for those who are unwell to get to a clinic, or arrange their day to accommodate a clinician to visit) then this may be increasing self-efficacy, reducing logistic challenges related to health service contact and thus reducing distress. In this clinical context of ongoing deterioration due to intra-thoracic cancer, our data do not support more than one session of supervised breathing training.

\section{Strengths and limitations}

In keeping with our research question, we did not have a 'usual care' control arm and cannot show the trajectory of outcome variables without any breathing intervention. We did not attempt to blind trial allocation from the research team. Others have attempted assessor blinding; advertent or inadvertent disclosure occurred in about half [10].

We have no objective measure of physical activity (e.g. walk test), aiming to maximize trial data completion with the most clinically relevant answers to the question, whilst minimizing participant burden. Repeat measures of performance and health status give a measure of everyday activity. Likewise, we made the decision not to document physiological measures of respiratory function such as peak expiratory flow rate or other comorbidities. By the time a patient with lung cancer becomes moderately or severely breathless, their prognosis is poor and symptom management is of paramount importance. Therefore, in order to focus on the most patient-relevant outcome, our primary outcome was patient-reported breathlessness, with other measures of breathlessness and performance status as secondary outcomes, rather than surrogate pathophysiological biomarkers. Both peak flow rate and the 6-minute walk test correlate poorly with quality of life and breathlessness in this complex setting [38]. Likewise, we have described our population (those who have had the treatment for their cancer and any other co-morbidities causing breathlessness optimized; refractory breathlessness) by symptom intensity and performance status rather than with co-morbidities. Potential participants with medical problems or comorbidities considered sufficient to affect trial completion were excluded. Eligibility criteria also required that identified reversible causes of breathlessness were treated prior to trial entry if appropriate to do so, in the opinion of the attending clinician.

\section{Generalizability}

The pragmatic nature of this trial is a major strength. It involved a variety of settings, centres, and clinicians across the UK. The results should therefore be generalizable across a wide range of service settings and delivery models. Furthermore, there are few trials of supportive 
Table 3 Primary analysis point (4 weeks)

\begin{tabular}{|c|c|c|c|c|c|c|}
\hline Variable & Three sessions & One session & Total & P-Value ${ }^{\dagger}$ & 95\% Confidence Interval & $\begin{array}{l}\text { Adjusted coefficient }{ }^{* *} \\
(95 \% \text { Confidence Interval) }\end{array}$ \\
\hline $\begin{array}{l}N \text { with sufficient NRS scores over } \\
4 \text { weeks to calculate AUC }\end{array}$ & 43 & 81 & 124 & & & \\
\hline \multicolumn{7}{|l|}{$\begin{array}{l}\text { AUC (Cubic) by Week } 4 \text { Breathlessness } \\
\text { NRS: mean (SD) }\end{array}$} \\
\hline Worst & $22 \cdot 86(7 \cdot 12)$ & $22 \cdot 58(7 \cdot 10)$ & $22 \cdot 68(7 \cdot 08)$ & $0 \cdot 83$ & $(-2 \cdot 31,2 \cdot 87)$ & $1 \cdot 05(-1 \cdot 64,3 \cdot 75)$ \\
\hline Average & $19 \cdot 45(6 \cdot 51)$ & $19 \cdot 14(6 \cdot 27)$ & $19 \cdot 24(6 \cdot 33)$ & $0 \cdot 79$ & $(-2 \cdot 00,2 \cdot 62)$ & $0 \cdot 81(-1 \cdot 48,3 \cdot 10)$ \\
\hline Distress* $(\mathrm{NHI}=42, \mathrm{NLI}=84)$ & $16 \cdot 23(7 \cdot 17)$ & $12 \cdot 29(8 \cdot 27)$ & $13 \cdot 60(8 \cdot 11)$ & $0 \cdot 01$ & $(0 \cdot 98,6 \cdot 91)$ & $3 \cdot 24(0 \cdot 25,6 \cdot 23)$ \\
\hline Cope & $25 \cdot 47(7.36)$ & $27.16(6.96)$ & $26.58(7.12)$ & 0.20 & $(-4.27,0.90)$ & $-0.87(-3.72,1.99)$ \\
\hline Care & $31.02(8.62)$ & $31.11(8.13)$ & $31.08(8.27)$ & 0.95 & $(-3.11,2.93)$ & $0.25(-2.78,3.28)$ \\
\hline $\begin{array}{l}\text { Chronic Respiratory Questionnaire -SAS } \\
\text { at Week 4: mean (SD) }\end{array}$ & $N=37$ & $N=71$ & $N=108$ & & & \\
\hline Dyspnoea domain & $4 \cdot 41(1 \cdot 33)$ & $4 \cdot 62(1 \cdot 43)$ & $4 \cdot 55(1 \cdot 39)$ & $0 \cdot 47$ & $(-0 \cdot 77,0 \cdot 35)$ & $-0 \cdot 38(-0 \cdot 89,0 \cdot 13)$ \\
\hline Fatigue & $3 \cdot 36(1 \cdot 05)$ & $3 \cdot 47(1 \cdot 25)$ & $3 \cdot 43(1 \cdot 18)$ & $0 \cdot 63$ & $(-0 \cdot 59,0 \cdot 36)$ & $0 \cdot 06(-0 \cdot 40,0 \cdot 51)$ \\
\hline Emotional Function & $4 \cdot 59(1 \cdot 08)$ & $4 \cdot 68(1 \cdot 13)$ & $4 \cdot 65(1 \cdot 11)$ & $0 \cdot 69$ & $(-0 \cdot 54,0 \cdot 36)$ & $0 \cdot 12(-0 \cdot 26,0 \cdot 51)$ \\
\hline Mastery & $4 \cdot 44(1 \cdot 25)$ & $5 \cdot 03(1 \cdot 15)$ & $4 \cdot 83(1 \cdot 21)$ & $0 \cdot 02$ & $(-1 \cdot 06,-0 \cdot 11)$ & $-0 \cdot 34(-0 \cdot 75,0 \cdot 07)$ \\
\hline
\end{tabular}

Descriptive statistics of the primary and secondary breathlessness outcome measures, P-values and unadjusted and adjusted confidence intervals for the difference between the two arms

SAS self administered survey (supported by research nurse); SD standard deviation; AUC area under the curve; NHI number in three sessions arm; NLI number in one-session arm

*adjusted for baseline breathlessness intensity, gender, smoking status and age

${ }^{\dagger} \mathrm{P}$-values based on student's test with equal variance assumed

Table 4 Descriptive statistics for the summary weekly measures for repeated measures

\begin{tabular}{|c|c|c|c|c|c|c|}
\hline \multirow[t]{3}{*}{ Measure } & \multicolumn{2}{|l|}{ Baseline } & \multicolumn{2}{|l|}{ Week 4} & \multicolumn{2}{|l|}{ Week 8} \\
\hline & \multicolumn{2}{|l|}{ mean; SD (N) } & \multicolumn{2}{|l|}{ mean; SD (N) } & \multicolumn{2}{|l|}{ mean; SD (N) } \\
\hline & Three sessions & Single session & Three sessions & Single session & Three sessions & Single session \\
\hline \multicolumn{7}{|c|}{ Breathlessness NRS scores } \\
\hline Worst & $6.7(2.0)[6]$ & $6.9(1.9)[12]$ & $6.1-2.4(37)$ & $5.7-2.4(70)$ & $6.2-2.4(25)$ & $5.8-2.6(56)$ \\
\hline Average & $5.4(1.8)[6]$ & $5.6(1.6)[13]$ & $5.3-2(37)$ & $4.9-2.3(70)$ & $5.2-2.2(26)$ & $4.8-2.3(56)$ \\
\hline Distress & $4.7(2.8)[7]$ & $4.6(3.1)[12]$ & 4.6-2.6 (37) & $4.1-3.0(70)$ & $3.8-2.7(25)$ & $3.5-3.1(56)$ \\
\hline Cope & $6.4(2.1)[6]$ & $7.0(2.1)[12]$ & $7-2.1(37)$ & $7.5-1.9(70)$ & $7.0-2.3(25)$ & $7.6-2.2(54)$ \\
\hline Satisfaction with care & $6.3(3.4)[9]$ & $6.5(3.4)[20]$ & $8.9-1.3(37)$ & $8.5-2.0(70)$ & $8.6-1.9(25)$ & $8.5-2.0(55)$ \\
\hline \multicolumn{7}{|l|}{ CIEQ-Chr } \\
\hline Injustice & $4.5-2.7(50)$ & $4.2-2.6(100)$ & $4-2.6(35)$ & $3.4-2.6(69)$ & 3.3-2.7 (26) & $3.2-2.2(55)$ \\
\hline Catastrophizing & $5.6-3.7(50)$ & $4.3-3.3(100)$ & $4.9-3.8(34)$ & $3.6-3.1(69)$ & $4.0-3.2(26)$ & $3.2-3.1(55)$ \\
\hline \multicolumn{7}{|c|}{ Chronic Respiratory Questionnaire - SAS: } \\
\hline Dyspnoea domain & $3.8-1.2(51)$ & $3.6-1.3(103)$ & $4-1.3(37)$ & $4.1-1.5(71)$ & $4.3-1.5(26)$ & $4.1-1.6(57)$ \\
\hline Fatigue & $3-0.95(51)$ & $3.3-1.1(102)$ & $3.4-1(37)$ & $3.5-1.1(71)$ & $3.6-1.3(26)$ & $3.5-1.4(57)$ \\
\hline Emotional functioning & 4.1.-1.1 (51) & $4.5-1.3(102)$ & $4.6-1.1(37)$ & $4.7-1.1(71)$ & $4.6-1.0(26)$ & $4.8-1.2(57)$ \\
\hline Mastery & $3.9-1.2(51)$ & $4.5-1.2(102)$ & $4.4-1.3(37)$ & $5.0-1.1(71)$ & $4.9-1.3(26)$ & $5.1-1.3(57)$ \\
\hline \multicolumn{7}{|l|}{ HADS score } \\
\hline Anxiety & $7.7-4.3(51)$ & $6.2-4.2(102)$ & $7.2-3.8(37)$ & $5.3-4.1(69)$ & $6.8-4.2(26)$ & $5.1-4.0(56)$ \\
\hline Depression & $6.6-3.0(51)$ & $6.2-3.9(102)$ & 5.8-3.7 (37) & $5.9-3.8(69)$ & $5.3-3.3(26)$ & $5.7-4.0(56)$ \\
\hline KPS & $72-13.5(65)$ & $71-9.9(95)$ & $71-9.0(47)$ & $68-12.8(51)$ & $73-9.6(26)$ & $73-8.9(35)$ \\
\hline
\end{tabular}

HADs, Hospital anxiety and depression scale; SAS, Self-administered survey (supported by research nurse); SD, Standard deviation; N, Number; CIEQ-Chr, Catastrophizing, injustice questionnaire in chronic disease; NRS, Numerical rating scale; KPS, Karnofsky Performance Scale 
and palliative care complex interventions with a costeffectiveness analysis; it is important that we can demonstrate that the reduced clinic time for participants allocated to the single session arm did not appear to need additional clinic time elsewhere in the health service.

\section{Clinical service implications}

This trial provides important data to inform service providers and commissioners. A single session prevents unnecessary burden for patients and carers and allows services to see more patients within current resources. Centres see hundreds of new lung cancer patients each year, but breathlessness services only see a fraction. Access to breathlessness clinics can be improved within current capacity; if services who currently offer more than one session change to a standard of single session breathing training, then more patients will receive this care.

\section{Conclusions}

There was no evidence that three sessions conferred additional benefits over one and it was not cost-effective. A single session therefore seems an appropriate way to provide this service. It should not be assumed that nonpharmacological supportive and palliative care interventions are without burden and assessment of this aspect should be incorporated in future supportive and palliative care research.

\section{Additional file}

Additional file 1: Post hoc sensitivity analysis adjusting for the clinically significant difference in baseline CRQ mastery domain and HADS anxiety.

\section{Abbreviations}

AUC: Area under the curve; CRQ-SAS: Chronic Respiratory Questionnaire-Selfadministered survey; EQVAS: EQ-visual analogue scale; HADS: Hospital Anxiety and Depression Scale; KPS: Karnofsky performance scale; NRS: Numerical rating scale; QALY: Quality Adjusted Life Year; SD: Standard deviation.

\section{Competing interests}

All authors have completed the Unified Competing Interest form at www.icmje.org/coi_disclosure.pdf (available on request from the corresponding author) and declare that (1) MJ, RB, SN, MK, DT have institutional support from the National Institute for Health Research, Research for Patient Benefit funding stream for the submitted work and AE's institution received NHS support costs from the clinical local research network; (2) MJ is a consultant (institutional funding) with Mayne Pharma that might have an interest in the submitted work. There are no other interests to declare.

\section{Authors' contributions}

$\mathrm{MJ}$ acts as guarantor for this trial and affirms that the manuscript is an honest, accurate, and transparent account of the trial being reported; that no important aspects of the trial have been omitted; and that any discrepancies from the trial as planned (and, if relevant, registered) have been explained. MJ conceived the trial; all authors contributed to the design; MK provided statistical expertise and GR provided health economic expertise. MK, GR, and MJ interpreted the data; MJ prepared the first draft of the manuscript and all authors contributed to important intellectual content, critical review, and approved the final draft.

\section{Acknowledgements}

With thanks to the patients who participated, the site investigators and clinical teams who supported the trial, and in particular the clinicians who delivered the trial intervention: Chris Maloney, Claire Myers, Clare Horne, Felicity Pryor (Physiotherapists) at East Kent University Hospitals NHS Trust; Lindsay Fitzpatrick and Dawn Beaty (Lung Cancer Clinical Nurse Specialists) at University Hospitals Coventry and Warwickshire NHS Trust; Anne English (Physiotherapy Clinical Specialist) at Hull and East Yorkshire Hospitals NHS Trust and Dove House Hospice, Hull and Marie D'Arcy (Lung Cancer Clinical Nurse Specialist) at Hull and East Yorkshire Hospitals NHS Trust; Catherine Moffat and Malika Harboun (Physiotherapists) and Julie Burkin (Occupationa Therapist) at Cambridge University Hospitals NHS Foundation Trust; Jennifer Sherburn (Physiotherapist) at Dartford and Gravesham NHS Trust; Phillippe Nicoll (Physiotherapist) at NHS Wales Cardiff and Vale University Health Board and Velindre NHS Trust; Lesley Somerville, (physiotherapist) at Greater Glasgow and Clyde NHS. Thanks also to Ms Annie Jones, our public and patient involvement representative member of the management group for invaluable advice.

\section{Funding}

This is a summary of independent research funded by the National Institute for Health Research, Research for Patient Benefit funding stream; grant reference number PB-PG-0609-19066. The views expressed are those of the authors and not necessarily of the NHS, the NIHR, or the Department of Health. The funders of the trial had no role in trial design, data collection, analysis or interpretations, or writing of the report. The corresponding author had full access to all the data and final responsibility for the decision to submit for publication.

\section{Data sharing}

Relevant anonymised patient level data available on reasonable request to the chief investigator.

\section{Role of the sponsor}

The sponsors did not have any role in the design, conduct, interpretation, review, approval, or control of this article.

\section{Author details}

${ }^{1}$ Hull York Medical School, Hertford Building, University of Hull, Hull HU6 7RX, UK. ${ }^{2}$ Department of Health Sciences, University of York, York, UK. ${ }^{3}$ Centre for Health Economics, University of York, York, UK. ${ }^{4}$ Department of Sport, Health and Exercise Science, University of Hull, Hull, UK. ${ }^{5}$ Dove House Hospice, Hull, UK. ${ }^{6}$ Humber NHS Foundation Trust, Willerby, UK. 'Castle Hill Hospital, Hull, UK. ${ }^{8}$ University of Cambridge, Cambridge, UK. ${ }^{9}$ Palliative Care Service, Cambridge University Hospitals NHS Trust, Cambridge, UK.

Received: 15 May 2015 Accepted: 14 August 2015

Published online: 07 September 2015

\section{References}

1. International Agency for Research on Cancer. GLOBOCAN 2008 (IARC). 2013. http://globocan.iarc.fr/Default.aspx. Accessed 24 ${ }^{\text {th }}$ August 2015

2. Muers MF, Round CE. Palliation of symptoms in non-small cell lung cancer: a study by the Yorkshire Regional Cancer Organisation Thoracic Group. Thorax. 1993:48:339-43.

3. Edmonds P, Higginson I, Altmann D, Sen-Gupta G, McDonnell M. Is the presence of dyspnea a risk factor for morbidity in cancer patients? J Pain Symptom Manage. 2000;19:15-22

4. Saracino A, Weiland TJ, Jolly B, Dent AW. Verbal dyspnoea score predicts emergency department departure status in patients with shortness of breath. Emerg Med Australas. 2010;22:21-9.

5. Zhao I, Yates P. Non-pharmacological interventions for breathlessness management in patients with lung cancer: a systematic review. Palliat Med. 2008:22:693-701.

6. Booth S, Farquhar M, Gysels M, Bausewein C, Higginson IJ. The impact of a breathlessness intervention service (BIS) on the lives of patients with intractable dyspnea: a qualitative phase 1 study. Palliat Support Care. 2006:4:287-93.

7. Bredin M, Corner J, Krishnasamy M, Plant H, Bailey C, A'Hern R. Multicentre randomised controlled trial of nursing intervention for breathlessness in patients with lung cancer. BMJ. 1999;318:901-4. 
8. Corner J, Plant H, A'Hern R, Bailey C. Non-pharmacological intervention for breathlessness in lung cancer. Palliat Med. 1996;10:299-305.

9. Yates P, Hardy J, Kwun F, Brunelli V, Zhao I, Clavarino I. A randomised controlled trial of a nonpharmacological intervention for dyspnoea. [Abstract] Clinical Oncological Society of Australia (COSA) 38th Annual Scientific Meeting, Perth, Western Australia. Asia-Pacific Journal of Clinical Oncology. Blackwell Publishing Asia, Australia 2011; 7:82

10. Farquhar MC, Prevost A, McCrone P, Brafman-Price B, Bentley A, Higginson $I$, et al. Is a specialist breathlessness service more effective and costeffective for patients with advanced cancer and their carers than standard care? Findings of a mixed-method randomised controlled trial. BMC Med. 2014;12:194

11. Higginson IJ, Bausewein C, Reilly C, Wei G, Gysels M, Dzingas M, et al. An integrated palliative and respiratory care service for patients with advanced disease and refractory breathlessness: a randomised controlled trial. Lancet Respir Med. 2014;2:979-87.

12. NICE. Guidance for the Diagnosis and Treatment of Lung Cancer. Methods, evidence \& guidance. London: National Collaborating Centre for Acute Care; 2005.

13. Spruit MA, Singh SJ, Garvey C, ZuWallack R, Nici L, Rochester C, et al. ATS/ ERS Task Force on Pulmonary Rehabilitation. An official American Thoracic Society/European Respiratory Society statement: key concepts and advances in pulmonary rehabilitation. Am J Respir Crit Care Med. 2013;188:e13-64.

14. Heran BS, Chen JM, Ebrahim S, Moxham T, Oldridge N, Rees K, et al. Exercise-based cardiac rehabilitation for coronary heart disease. Cochrane Database Syst Rev. 2011;7, CD001800.

15. Granger CL, McDonald CF, Berney S, Chao C, Denehy L. Exercise intervention to improve exercise capacity and health related quality of life for patients with non-small cell lung cancer: a systematic review. Lung Cancer. 2011;72:139-53.

16. Johnson M, Moore S. Research into practice: the reality of implementing a non-pharmacological breathlessness intervention into clinical practice. Eur J Oncol Nurs. 2003;7:33-8.

17. Booth S, Bausewein C, Higginson I, Moosavi SH. Pharmacological treatment of refractory breathlessness. Expert Rev Respir Med. 2009;3:21-36.

18. Barton R, English A, Nabb S, Rigby AS, Johnson MJ. A randomised trial of high vs low intensity training in breathing techniques for breathless patients with malignant lung disease: a feasibility study. Lung Cancer. 2010;70:313-9.

19. Wilcock A, Crosby V, Clarke D, Tattersfield A. Repeatability of breathlessness measurements in cancer patients. Thorax. 1999;54:375.

20. Gift AG, Narsavage G. Validity of the numeric rating scale as a measure of dyspnea. Am J Crit Care. 1998;7:200-4.

21. Schunemann HJ, Goldstein R, Mador MJ, McKim D, Stahl E, Puhan M, et al. A randomised trial to evaluate the self-administered standardised chronic respiratory questionnaire. Eur Respir J. 2005;25:31-40.

22. Zigmond AS, Snaith RP. The hospital anxiety and depression scale. Acta Psychiatr Scand. 1983;67:361-70.

23. Karnofsky DA, Burchenal JH. The clinical evaluation of chemotherapeutic agents in cancer. In: MacLeod CC, editor. Evaluation of chemotherapeutic agents in cancer. New York: Columbia University Press; 1949. p. 191-205.

24. Kind P. The EuroQoL instrument: an index of health-related quality of life. In: Spilker B, editor. Quality of life and pharmacoeconomics in clinical trials. Philadelphia: Lippincott-Raven; 1996.

25. Carver CS. You want to measure coping but your protocol's too long: consider the brief COPE. Int J Behav Med. 1997;4:92-100.

26. John OP. Big five trait taxonomy. In: Pervin LA, John OP, editors. Handbook of Theory and Research. 2nd ed. New York, NY: The Guilford Press; 2001.

27. Kahan BC, Morris TP. Adjusting for multiple prognostic factors in the analysis of randomised trials. BMC Med Res Methodol. 2013;13:99.

28. Johnson MJ, Bland JM, Oxberry SG, Abernethy AP, Currow DC. Clinically important differences in the intensity of chronic refractory breathlessness. J Pain Symptom Manage. 2013:46:957-63.

29. Lansing RW, Gracely RH, Banzett RB. The multiple dimensions of dyspnea: review and hypotheses. Respir Physiol Neurobiol. 2009;167:53-60.

30. Parshall MB, Meek PM, Sklar D, Alcock J, Bittner P. Test-retest reliability of multidimensional dyspnea profile recall ratings in the emergency department: a prospective, longitudinal study. BMC Emerg Med. 2012;12:6.

31. von Leupoldt A, Ambruzsova R, Nordmeyer S, Jeske N, Dahme B. Sensory and affective aspects of dyspnea contribute differentially to the Borg scale's measurement of dyspnea. Respiration. 2006;73:762-8.
32. Currow DC, Smith J, Davidson PM, Newton PJ, Agar MR, Abernethy AP. Do the trajectories of dyspnea differ in prevalence and intensity by diagnosis at the end of life? A consecutive cohort study. J Pain Symptom Manage. 2010;39:680-90.

33. Li J, Girgis A. Supportive care needs: are patients with lung cancer a neglected population? Psychooncology. 2006;15:509-16.

34. Rueda JR, Sola I, Pascual A, Subirana CM. Non-invasive interventions for improving well-being and quality of life in patients with lung cancer. Cochrane Database Syst Rev. 2011;9, CD004282.

35. Morrison RS, Penrod JD, Cassel JB, Caust-Ellenbogen M, Litke A, Spragens L, et al. Cost savings associated with US hospital palliative care consultation programs. Arch Intern Med. 2008;168:1783-90.

36. Temel JS, Greer JA, Muzikansky A, Gallagher ER, Admane S, Jackson VA, et al. Early palliative care for patients with metastatic non-small-cell lung cancer. N Engl J Med. 2010;363:733-42.

37. Kutikova L, Bowman L, Chang S, Long SR, Obasaju C, Crown WH. The economic burden of lung cancer and the associated costs of treatment failure in the United States. Lung Cancer. 2005;50:143-54.

38. de Torres JP, Pinto-Plata V, Ingenito E, Bagley P, Gray A, Berger R, et al. Power of outcome measurements to detect clinically significant changes in pulmonary rehabilitation of patients with COPD. Chest. 2002;121:1092-8.

39. Blagbrough M, Coulthard M. The Calming Hand. Oxford Textbook of Palliative Medicine. 4th ed. Oxford: Oxford University Press; 2010.

\section{Submit your next manuscript to BioMed Central and take full advantage of:}

- Convenient online submission

- Thorough peer review

- No space constraints or color figure charges

- Immediate publication on acceptance

- Inclusion in PubMed, CAS, Scopus and Google Scholar

- Research which is freely available for redistribution 